\title{
Passive Sentences in Jordanian Arabic underthe Minimalist Approach
}

\author{
Dr. Islam M. Al-Momani \\ Princess Alia University College \\ Al-Balqa' Applied University (Jordan) \\ almomani_islam@yahoo.com
}

\author{
Naji Masned AlQbailat \\ Director of the Language Center \\ Al-Balqa' Applied University (Jordan) \\ najimq1@bau.edu.jo
}

\begin{abstract}
The paperaims at investigating the properties of passive sentences in Jordanian Arabic (henceforth) $J A$, and providing a deeper understanding and precise description of the syntactic and semantic features of passivization. It also aims at examining the structure and derivation of passive sentences under the Minimalist Program. Principles like feature checking, and merger are agued to provide an account for JA passive sentences. In case of active transitive sentences, the paper has claimed that JA has a strong NP feature of T which induces overt NP movement of the subject NP out of the VP internal position, that is, the [Spec, and VP]. In the passive transforms of transitive sentences, the object NP moves to the [Spec, VP], and then to the [Spec, IP] to have its Nom Case checked off. JA allows the merger between the passive morpheme and the verb. Thus, the verb raises to $T$ to have its $\varphi$ features checked by the $V$-features of $T$. Similar observations can be noticed in intransitive sentences, the subject NP must be fronted to [Spec, TP] in order to satisfy EPP features. The [+V] feature of $T$ attracts the verb to raise and check the $[+V]$ feature.
\end{abstract}

Keywords : Jordanian Arabic, passivization, movement, derivation, feature checking, merger, argument.

\section{INTRODUCTION}

Jordanian Arabic (JA) is one of the dialects of Modern Standard Arabic (MSA). It is a colloquial form which is spoken in Jordan by about eight million speakers, and is understood by most of the Arabs in the Arab world. The paper aims at introducing some grammatical features of passive sentences in JA. Both VSO, and SVO orders are observed in JA, but the focus of this paper is made on the latter which is preferred.

Before going into any details concerning passivization in JA, this section provides a brief analysis of the structure and derivation of passivization in the literature of modern linguistics. The point of focus will be on the different syntactic analyses concerning the availability of movement of the passive verb (movement/base generation). It also explains the view related to the raising of the object NP to the subject NP position.

The analysis of passivization has always been a topic of controversial debate in the literature of modern linguistics. Chomsky in his GB (1981) argues that passive sentences are characterized by external $\Theta$ role and case assignment. An external argument does not receive a $\Theta$ role (suppression of an external $\Theta$ role), and a complement does not receive Case within VP (absorption of an objective Case). Consider the following English example in (A), and its underlying structure in (B):

A. the wall was painted by John.

B. [e [ was [painted the wall [by John]]]]

The base verb 'paint' selects two NPs and assigns two $\Theta$ roles to them (Agent and Theme), but according to Chomsky's hypothesis, the passive morpheme 'en' suppresses the external $\Theta$ role (Agent) that the verb assigns to the subject NP, and absorbs the Acc. Case that the verb assigns to the object NP (Theme). As a consequence, the object NP must move to the subject position where it receives Nom. Case from 'Infl', (Inflection)', and the suppressed Agent NP, John optionally appears in an adjunct by phrase.

Jaeggli (1986) proposes that the properties of passivization are accounted for as in the following way: The passive receives external theta role of the predicate so that no longer assigned to [NP, S] position. 
Thus, the subject position is not assigned a thematic role in passive sentences. The second property, the passive suffix is assigned the accusative case so that an $[\mathrm{NP}]$ in the object position can no longer be assigned a structural case by the verb. Therefore, the object must move to the subject position to receive NOM-Case in passives.

Baker (1988) and Baker, Johnson \& Roberts (1989) examine passives that occur with an accusative verb based on Jaeggli analysis of the passive morphemes. Burzio (1986) observes the following correlation between ACC-Case assignment. His generalization is as the following:

I. A verb which lacks an external argument fails to assign Acc-Case.

II. A verb which fails to assign Acc-case fails to theta mark an externalargument.

There has been some attention given in the literature to movement of passive verbs. Proposals along this side have been made in the literature. In Baker, Johnson, and Robert (1989), for example, the passive morpheme -en is generated in 'Infl' as an argument. The main verb raises to 'Infl' and assign case to -en, triggering movement of the object NP to Spec, IP.

The majority of research has focused on 'long' movement, specifically $\mathrm{V}$ to $\mathrm{T}$ or 'Infl' and 'Infl' to 'C' as in the works of Vikner (1991, and 1995), Chomsky (1991), and Koopman (1984). 'Short' movement did not begin until the introduction of the VP internal subject hypothesis (Koopman and Sportiche 1988, 1991). This hypothesis proposes that the external arguments are generated in a specific position either inside the main VP or in the extended projection of the VP. This extended projection has been referred differently by different linguists, for example, Kratzer (1994) names it 'voi', Bowers (1993) names it Pr, Collins (1997) names it 'Tr', and Chomsky (1995) names it 'v'. The main idea in this hypothesis that the verb moves out of the $\mathrm{V}$ position either overtly or at LF, and raises to the head position of the extended projection.

A second type of 'short' movement discussed in the literature of the Generative Grammar is based on the theory which assumes that the component parts, i.e., meanings of a verb are built via operations which take place at the syntactic level (e.g., Collins and Thráinsson 1996), or at post lexical level which is governed by the same principles such as ECP/Head Movement Constraints as the syntactic level (e.g., Hale and Keyser 1993). This approach is referred to as Lexical Decomposition (LD). LD dates back to the days of generative semantics (e.g., Lakoff 1970).

\section{OBJECTIVES AND METHODOLOGY}

This paper aims at investigating passivization in JA. It aims at answering the following research questions:

a. How does passivization operate in JA?

b. What is the base position of the object NP in JA, and where does it move?

c. Is the object NP movement optional or obligatory?

d. Can the aspects of passive sentences in JA be straightforwardly accounted for in the light of derivation and feature-checking principles of the Minimalist Program?

The researchers follow the descriptive-analytical approach. The data used here is an amalgamation of genuine sentences uttered by native speakers of Jordanian Arabic. The following symbols are used to represent certain sounds in JA that do not match the IPA symbols:

$/ t ̦ /$ voic eless dental emphatic stop

/ș/ voiceless alveolar emphatic fricative

$/ \dot{\mathrm{g}} /$ voiced velar fricative

$/ \mathrm{h} /$ voiceless pharyngeal fricative

/ç/ voiced pharyngeal fricative

\section{DISCUSSION}

\section{Passivization of Transitive Sentences}

The passive construction in JA is expressed by a single but morphologically complex verb that is the verb stem appears to combine with the morpheme. Accordingly, the past passive sentences in JA are expressed by attaching the morpheme '?it' to the verb stem as in the following sentences: 
1. at-tufa hah ?it-akala-t

The-apple was- eaten-FM

The apple was eaten.

2. l-mansaf ?it-akal

The-mansaf was-eaten

Mansaf (kind of food) was eaten.

The present passive sentences in JA are expressed by attaching the morpheme 'bit' to the verb stem as in the following sentences

3. attufahah bit-t-akal

The-apple is-FM- eaten

The apple was eaten.

4. 1-mansaf bit-akal

The-mansaf is-eaten

Mansaf (kind of food) is eaten.

The passive in JA is an optional operation that deletes the subject NP, moves cyclically the object NP from its base position as the DP of VP to the [Spec, VP], then to the [Spec. IP]. The verb moves to T to check the $[+\mathrm{V}]$ feature, and merges with the passive morpheme "?it". This operation could be stated in (5):

5. NP $\quad \mathrm{V} \quad \mathrm{NP} \rightarrow$ (optional)

$\begin{array}{lll}1 & 2 & 3 \\ 3 & ? \text { it }+2 & \varnothing\end{array}$

To illustrate formula (5), consider the active sentences in (6), and their passive transformation in (7).
6. a. Aћmad
baç s-sayara
Aћmad
sold the-car

Ahmad sold the car.

6. b. Hind gasla-t 1- şћoon

Hind washed-FM the-dishes

Hind washed the dishes.
6. c. Aћmad
kasar
1-qazaz
Aћmad
broke
the-glass

Ahmad broke the glass.

7. a. s-sayara ?in-baçi-t

The-car was-sold-FM

The car was sold.

7. b. 1- șћoon ?it-gasal-in

The- dishes was-washed-PFM

The dishes were washed.

7. c. l-qazaz ?it-kassar

The-glass was broken

The glass was broken. 
Some sentences may meet the structural description of passivization. However, the passive transformation could be wrongly applied to transitive sentences. Consider the examples in (10), and their passive transformation in (11):

$\begin{array}{rll}\text { 8. a. Aћmad } & \text { ?iðakkar } & \text { Hind } \\ \text { Aћmad } & \text { remembered } & \text { Hind }\end{array}$

Ahmad remembered Hind.

8. b- Aћmad zaçal Hind

Ahmad offended Hind

Ahmad offended Hind.

9. a- *Hind ?it-ðakkara-t

Hind was-remembered-FM

Hind was remembered.

9. b- *Hind ?it-zaçala-t

Hind was-offended-FM

Hind was offended.

The passive sentence in JA does not express an agent. The subject of an active sentence is deleted when the sentence is passivized; whereas, the subject is kept when the sentence is not passivized. This means that the when the subject noun phrase of the verb undergoes the passive transformation is deleted, and leaves an empty node that has to be filled. The object noun phrase moves into the empty subject position producing the passive sentence.

Accordingly, verbs that undergo passive transformation could be subcategorized for the feature $+\mathrm{V}\left[\mathrm{VP} \_\right.$NP]. The intransitive verbs could be subcategorized for the feature +V[VP__PP]. Subgategorizational rules are lexical rules meant only for the operation of lexical insertion rules. Since phrase structure rules applies before lexical insertion rules, the subject node will be generated as a pre-terminal node that may or may not be filled by a lexical item. When the lexical rules apply to insert a noun phrase in the subject position of a verb which optionally undergoes passive transformation, we get an active sentence. When lexical insertion rules do not apply to insert a noun phrase in the subject position of such a verb, we get a sentence with an empty subject node that needs to be filled. This empty subject node triggers the passive transformation to move the object to fill the subject node, and thus generates a passive sentence.

Therefore, the passive transformation will be as the following:

In transitive sentences: the input to the passive transformation in JA is as in (10):

10. $[\varnothing \mathrm{V} \quad \mathrm{NP}]$

Showing its Structural Description and Structural Change, The passive transformation must be formulated as (11):

11. SD: $\varnothing \quad \mathrm{V}$ NP

$$
\begin{array}{rrrr}
1 & 2 & 3 & \\
& \text { SC } & 3 & ? \text { it }+2
\end{array}
$$

In order to decide the base positions of the verb, and the subject NP in active constructions in JA, consider the sentencesin (12) representing the VS order and the sentences in (13) representing the SV order, and examine their representation in (14), and (15).

$\begin{array}{lll}\text { 12. a-sarag-at } & \text { l-banaat } & \text { 1-mașaari } \\ \text { sarag-3fs } & \text { the-girls } & \text { the-money }\end{array}$

The girls stole the money. 


12. b- *sarag-in l-banaat l-maşaari

sarag-3fp-nom the-girls the-money

The girls stole the money.

13. a- 1-banaat sarag-in 1-maşaari

The-girlssarag-3fp-nom the-money

The girls stole the money.

13. b- *l-banaat sarag-at 1-maşaari

The-girls sarag-3fs- the-money

The girls stole the money.

(12a) is a grammatical one because the number agreement is spelled-out by the lexical subject '1banaat' only; whereas, (12b) is ungrammatical because the number agreement on the verb is spelledout by both the number suffix 'in' and the merged NP.Following Benmamoun (2000), the absence of the number suffix on the verb in VS order is due to the merger of the verb and the subject in the PF component. He assumes that the subject and the verb merge in the PF interface to form one single unit. Accordingly, the subject NP spells-out the number feature, and thus makes the presence of the number suffix redundant.

(13a) is grammatical because the $\mathrm{T}$ has strong $\mathrm{N}$-features in $\mathrm{SV}$ order. Therefore, there must be agreement according to number between the subject NP 'l-bannat' and the third feminine plural suffix 'in' attached to the verb 'sarag'. Lack of agreement according to number between the subject NP and the verb leads to the ungrammaticality of (13b).

Mobaidin (2002) proposes an analysis to subject verb agreement in standard Arabic, which is adopted by the researchers in this paper to explain the difference between the VS and SV orders in JA. Consider the structure in (14) that best represents (12a).

14.

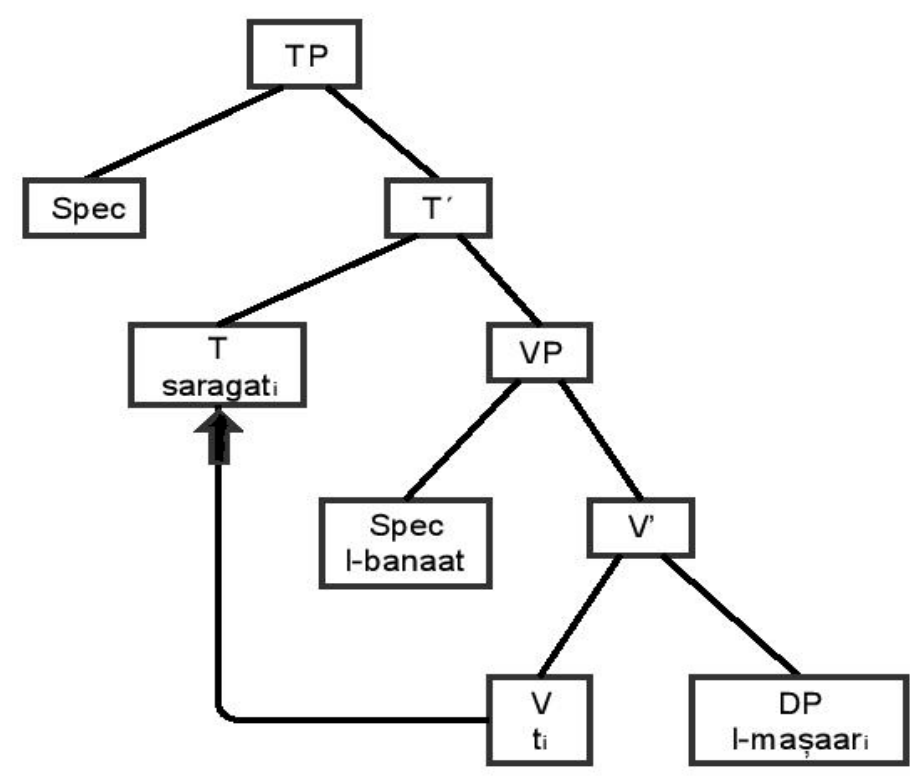

Mobaidin (2002:44) assumes that the T in VS order has weak N-features, and thus it is allowed to merge with a VP whose head is not inflected for number. Because there is no overt number suffix on the verb, the subject has to wait until after spell-out to check N-features of T. Accordingly, T in (14) has no number features since it fails to attract the subject NP '1-banaat' to [Spec, TP].

Whereas, in SV order as in structure (15) below, the Thas strong $\mathrm{N}$-features, and thus merges with the $\mathrm{VP}$ whose head is fully inflected. Therefore, the verb 'saragin' raises to $\mathrm{T}$ to check the V-features of $\mathrm{T}$ including number. Because $\mathrm{T}$ has strong $\mathrm{N}$-features, the subject $\mathrm{NP}$ 'l-banaat' raises up overtly to $[$ Spec, TP] to check its $\varphi$ features against those of $\mathrm{T}$. 
15.

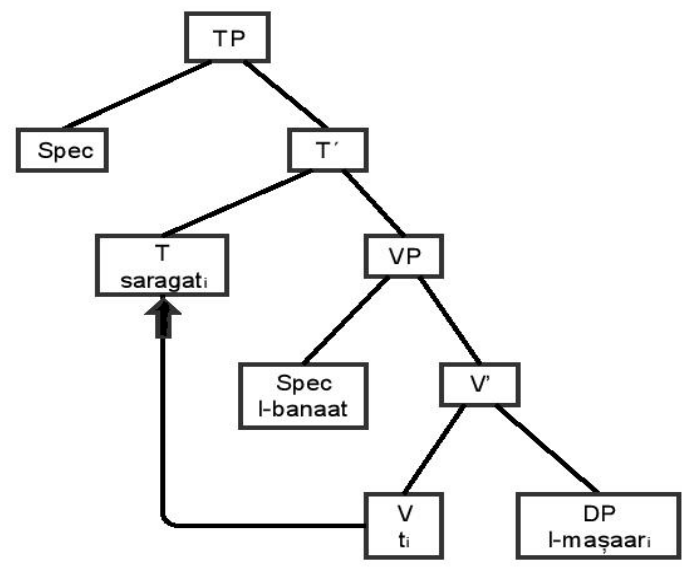

Following Chomsky (1993), a distinction between strong and weak features is made. Strong features namely, Case and $\varphi$ features that motivate overt syntactic movement of a verb and an NP must be checked before SPELL-OUT because they are assumed to be visible at PF. Weak features (Case and $\varphi$ features) which do not motivate overt syntactic movement but only covert LF movement are invisible at PF so they need not be checked off before SPELL-OUT. Thus, they are checked of at LF after SPELL-OUT.

Based on the aforementioned analyses, it could be assumed that theSV order in JA has strong Nfeatures of T which induces overt NP movement of the subject NP out of the VP internal position, that is, the [Spec, VP] to have its Nominative Case checked by the NP feature (Nominative Case feature) of $\mathrm{T}$ adjoined to TP satisfying the Extended Projection Principle (EPP). The verb overtly raises to $\mathrm{T}$ to have its $\varphi$ features checked by the $\mathrm{V}$ - features of $\mathrm{T}$.

The object NP 'l-mașaari' remains in its situ position and its Acc. Case is checked at LF where it enters into a checking relation with the verb which sustains its Acc. Case feature.

When the object NP is promoted to the subject position by passivization, and gets case, it is positioned either in [Spec, VP] or in [Spec, TP]. To examine this, consider the passive sentencesin (16), and (17), and their representationsare shown as in (18), and (19) respectively.

16. ?it-sarag-in l-mașaari

Were-stolen- PFM the-money

The money was stolen

17. l-maşaari ?it-sarag-in

The-money were-stolen-PFM

The money was stolen.

The researchers argue that in structure (18), the case of passive constructions in VS order, the verb raises overtly to ' $T$ ', and the object NP moves only to the [Spec, VP], only.

18.

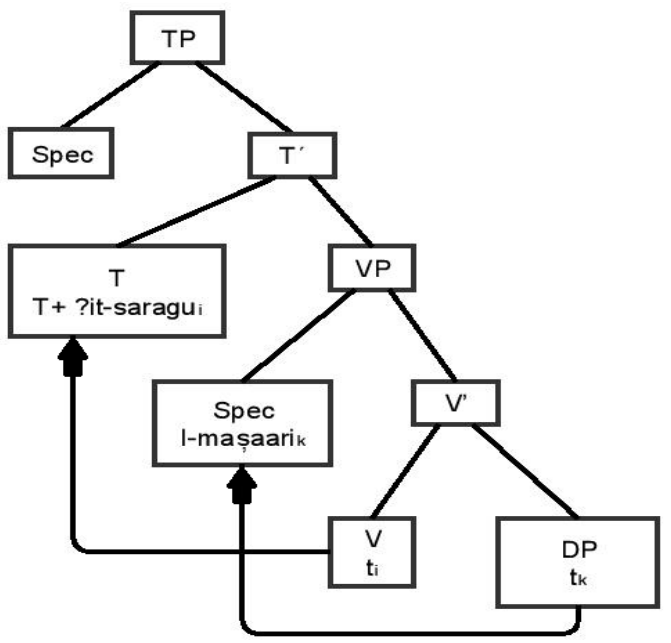


Chomsky (1995) suggests that movement is driven by morphological factors, and thus verbs raise to license or check their morphology. Given that passive verbs are marked, it is the passive verb that raises overtly. T attracts the verb to raise and check the $V$ features. Thus, The passive verb '?itsaragin' raises to $T$ and to have its $\varphi$ features checked by the $V$ - feature of $T$.

The direct object 'l-maşaari' in (18) moves directly to the [Spec, VP] before spell-out. The T which has an EPP feature that needs to be checked attracts the direct object, the closest element, to its checking domain.Because the case feature and $\varphi$ features of the nominative object NP '1-mașaari' enter into a checking relation with T, 'l-mașaari' behaves like an ordinary subject as it is marked with Nom. Case and induces subject-verb agreement.

In the case of SV order as in structure (19), the verb raises overtly to ' $T$ ', and the object NP moves cyclically to [Spec, VP], and then to [Spec, TP].

19.

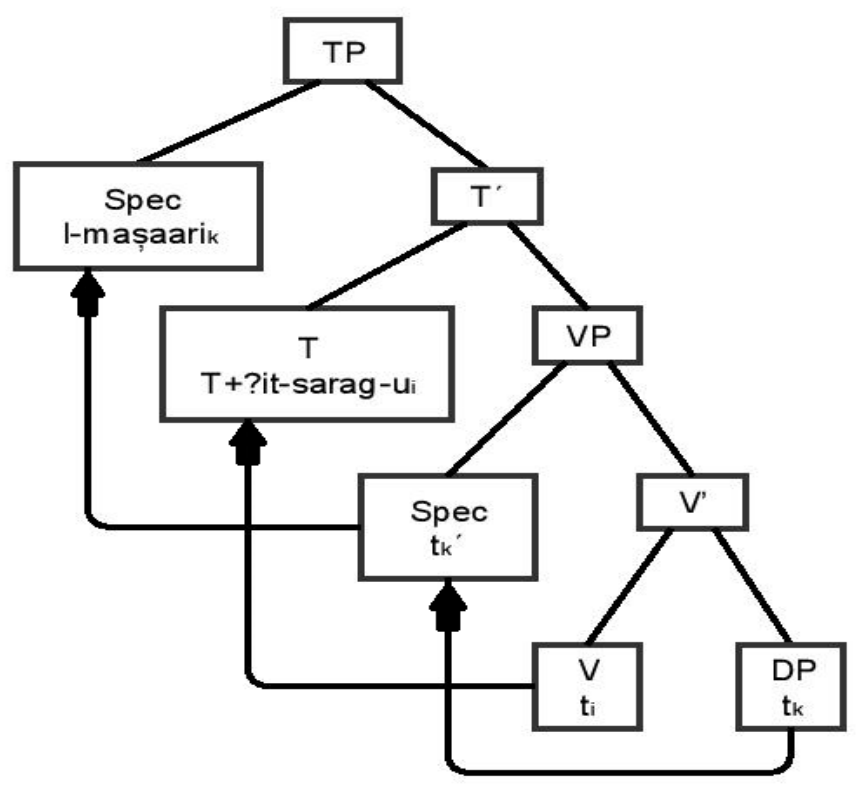

In (19), the object NP, 'l-mașaari' moves to the [Spec, VP], and then to the [Spec, IP] to have its Nom Case checked off. The $\mathrm{T}$ attracts the verb to raise and check the $\mathrm{V}$-features. Thus, The passive verb '? itsaragin' raises to $\mathrm{T}$ to have its $\varphi$ features checked by the $\mathrm{V}$ - features of $\mathrm{T}$.

\section{Pas sivization of Intransitive Sentences}

To complicate things in JA, passive applies to intransitive sentences. The intransitive sentences in (20) have passive transforms in (21):

20. a- 1-muddeer

The-manager ça

on 1-țtalab

the-request

The manager agreed on the request.

20. b- Aћmad

đitik

çala

Hind

Aћmad laughed on

Hind

Ahmad laughed at Hind.

20. c- Aћmad

$$
\text { lçib }
$$

Aћmad

played

bi

1-radio

Ahmad played with the radio

21. a- 1-țtalab

?it-waafag

çaley-h

The-request was-agreed

on-it

The request was agreed on. 


\begin{tabular}{lll}
\hline 21. b- Hind & ?it-đaћak & çaley-ha \\
Hind & was-laughed & at-her
\end{tabular}

Hind was laughed at.

21. c- 1-radio ?it-lçab fii-h

The-radio was-played in-it

The radio was played with.

The representation of the active sentence in (20 a) is as shown in (22).

22.

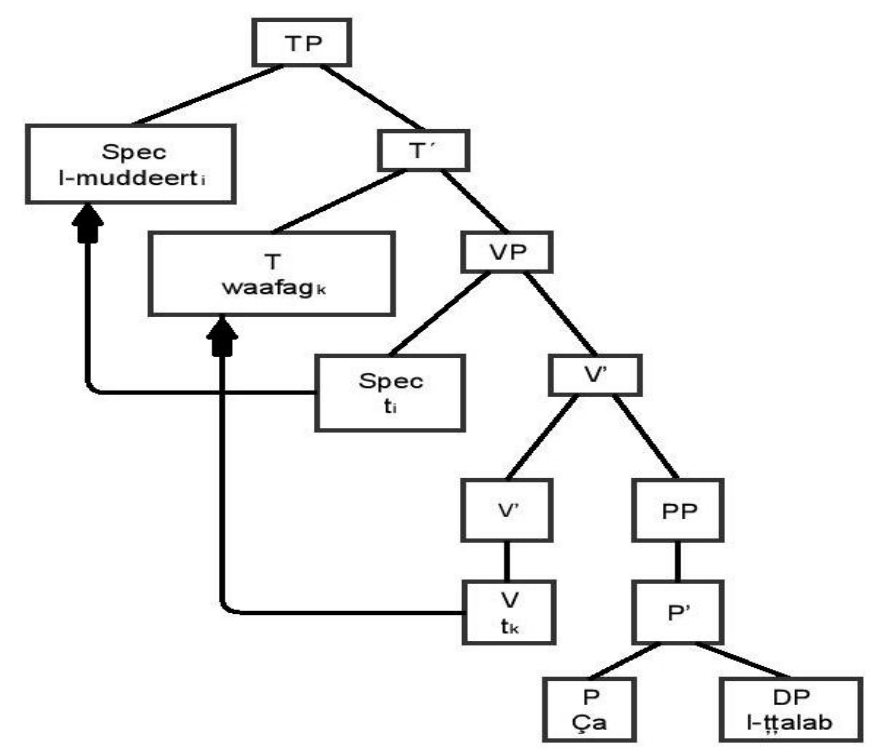

Similar observation can be noticed here, the subject NP, 'l-muddeer' must be fronted to [Spec, TP] in order to satisfy EPP features. The $[+\mathrm{V}]$ feature of $\mathrm{T}$ attracts the verb, 'waafag' to raise and check the $[+\mathrm{V}]$ feature.

The same analysis is appropriate for the passive sentence in (21 a) which is represented as in the tree diagram in (23).

23.

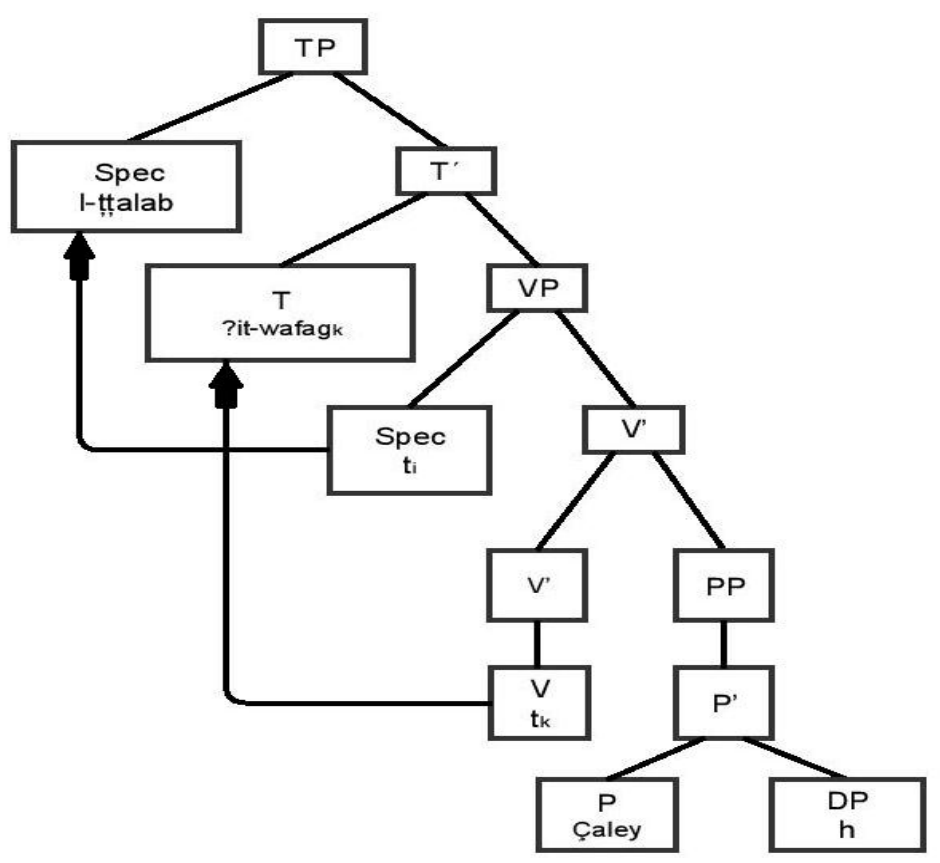


Although sentences in (21) show that the passive transformation of intransitive sentences is very productive in JA, most intransitive sentences do not passivize. The sentences in (24) have the same underlying structures as in (20), but applying the same rule that applies to (20) would result in producing ungrammatical sentences as in (25):

24. a- Aћmad tmasxar çla Hind

Aћmad noised on Hind

Ahmad disturbed Hind.

24. b- Aћmad tşşannat çla Hind

Aћmad eavesdropped on Hind

Ahmad eavesdropped on Hind.

$\begin{array}{rrr}\text { 25. a- *Hind } & \text { ?it-tmasxar } & \text { çlee-ha } \\ \text { Hind } & \text { was-noised } & \text { on-her }\end{array}$

Hind was disturbed.

$\begin{array}{rll}\text { 25. b- *Hind } & \text { ?it- ţ̦şannat } & \text { çlee-ha } \\ \text { Hind } & \text { was- eavesdropped } & \text { on-her }\end{array}$

Hind was eavesdropped on.

It could be concluded here that the input to the passive transformation of intransitive sentences in JA is as in (26):

26. $\left[\varnothing \quad\left[\begin{array}{lll}\varnothing & \mathrm{V} & \mathrm{P}\end{array}\right]\right.$

Showing its Structural Description and Structural Change, The passive transformation must be formulated as in (27):

$\begin{array}{clll}\text { 27. SD: } \varnothing & \text { V } & \text { P } & \text { NP } \\ 1 & 2 & 3 & 4 \\ \text { SC: NP } & ? i t+2 & 3 & \text { Pro. }\end{array}$

\section{CONCLUSiON}

The paper has investigated the derivation, features and characteristics of passive sentences in JA. After exposing the structure of passivization in JA to the claim of the Minimalist Program, it has been found that a lot of its aspects can be straightforwardly accounted for in the light of feature-checking and merger principles. In case of active transitive sentences, the paper has claimed that JA has a strong NP feature of T which induces overt NP movement of the subject NP out of the VP internal position, that is, the [Spec, VP]. In the passive transforms of transitive sentences the object NP moves to the [Spec, VP], and then to the [Spec, IP] to have its Nom Case checked off. JA allows the merger between the passive morpheme '? it' and the verb. Thus, the verb raises to $T$ to have its $\varphi$ features checked by the V-features of T. Similar observations can be noticed in intransitive sentences, the subject NP must be fronted to [Spec, TP] in order to satisfy EPP features. The $[+\mathrm{V}]$ feature of $\mathrm{T}$ attracts the verb to raise and check the $[+\mathrm{V}]$ feature.

\section{REFERENCES}

Baker, M. (1988a). Incorporation: A Theory of Grammatical Function Changing. Chicago Press.

Baker, M. Johnson, K. and Robert, I. (1989). Paper in Passive Arguments. Linguistics Inquiry 20, 219-251.

Benmamoun, E. (2000). The Feature Structure of Functional Categories: A Comparative Study of Arabic Dialects. Oxford: Oxford University Press.

Bowers, J. (1993). The Syntax of Predication. Linguistics Inquiry24,159-656.

Burzio, L. (1986). Italian syntax: A Government-Binding Approach. Dordrecht: Reidel.

Chomsky, N. (1995). The Minimalist Program. Cambridge, Massachusetts: MIT Press. 
Chomsky, N. (1993). A Minimalist Program for Linguistic Theory. In TheViewfrom Building20: Essays in Linguistics in Honor of Sylvain Bromberger, ed. Kenneth Hale and Samuel jay Keyser, 1-52. Cambridge, Massachusetts: MIT Press.

Chomsky, N. (1991). Some Notes on Economy of Derivation and Representation. In Principles and Parameters in Comparative Grammar, ed. Robert Freidin, 417-454. Cambridge, Massachusetts: MIT Press.

Chomsky, N. (1981). Lectures on Government and Binding. Dordrecht: Foris.

Collins, C. and Thráinsson, H. (1996). VP- internal structure and object shift in Icelandic. Linguistic Inquiry 27, 391-444.

Hale, K and Keyser, S. (1993). On Argument Structure and Lexical Expression of Syntactic Relations. In The View from Building 20: Essays in Linguistics in Honor of Sylvain Bromberger, ed. Kenneth Hale and Samuel Jay Keyser, 43-109. Cambridge, Mass.: MIT Press.

Jaeggli, O. (1986). Passive. Linguistic Inquiry17, 587-622.

Kratzer, A. (1994). On exrenal argument. Occasional Paper 17, University of Massachusetts, Amherst.

Koopman, H. (1984). The Syntax of Verbs. Dordrecht: Foris.

Koopman, H and Sportiche, D. (1988). Subjects. Ms., UCLA, Los Angeles, California.

Koopman, H and Sportiche, D. 1991. The Position of Subjects. Lingua85, 211-258.

Lakoff, G. (1970). Irregularity in Syntax. New York: Holt, Rinehart and Winston.

Mobaidin, H. (20000). Basic Word Order in Arabic: A Minimalist Account. Mu'tah Lil-Buhuuth wadDiraasaat 17, 35-56. 